\title{
Leukotrienes Increase Blood-Brain Barrier Permeability Following Intraparenchymal Injections in Rats
}

\author{
Keith L. Black, MD, and Julian T. Hoff, MD
}

\begin{abstract}
To examine whether leukotrienes could increase blood-brain barrier permeability, rats were anesthetized and injected intravenously with Evans blue. Ten microliters of vehicle, of leukotrienes $B_{4}, C_{4}$, or $E_{4}$, or of arachidonic acid was injected over 1 hour directly into the brain parenchyma. The percentage of the total surface area of Evans blue extravasation in a coronal section of brain centered on the injection site was then determined as an estimate of bloodbrain barrier permeability. Leukotrienes $\mathbf{B}_{4}, \mathbf{C}_{4}$, and $\mathbf{E}_{4}$, and arachidonic acid all increased blood-brain barrier permeability, but this effect was lost when the total dose was reduced to $20 \mathrm{ng}$. Increased blood-brain barrier permeability induced by arachidonic acid could be prevented by pretreatment with the lipoxygenase inhibitor BW755C, but not with indomethacin. Leukotrienes may play a role in the development of increased blood-brain barrier permeability after cerebral injury.
\end{abstract}

Black KL, Hoff JT: Leukotrienes increase blood-brain barrier permeability following intraparenchymal injections in rats. Ann Neurol 18:349-351, 1985

Arachidonic acid is metabolized by means of two pathways: cyclooxygenase, leading to the formation of prostaglandins, and lipoxygenase, leading to the production of leukotrienes. Prostaglandins have been implicated in the pathogenesis of cerebral ischemia. This is in part supported by studies showing that cerebral blood flow in ischemic brain is increased after treatment with cyclooxygenase inhibitors and prostacyclin [3]. Leukotrienes have been shown to increase vascular permeability in postcapillary venules, increase blood flow, and elicit erythema and wheal formation in skin [10]. Leukotriene concentrations are reportedly increased in cerebral ischemia [7], and we recently noted that the injection of leukotriene $\mathrm{C}_{4}$ directly into brain parenchyma increased blood-brain barrier (BBB) permeability [2]. Consequently, leukotrienes may play a role in the pathogenesis of vasogenic cerebral edema $[2,3,7]$.

This study was designed to determine (1) whether intraparenchymal injections of leukotriene (LT) $\mathrm{B}_{4}$, $\mathrm{LTC}_{4}$, or $\mathrm{LTE}_{4}$, or arachidonic acid increase BBB permeability; and (2) whether the lipoxygenase inhibitor BW755C [3-amino-1-(3-(trifluoromethylphenyl)-2-pyrazoline $[8,9]$ prevents the increased BBB permeability induced by arachidonic acid.

\section{Materials and Methods}

Wistar rats weighing 250 to $350 \mathrm{gm}$ were anesthetized with pentobarbital ( $40 \mathrm{mg}$ per kilogram of body weight) injected intraperitoneally. PE-10 polyethylene catheters were inserted into the femoral artery and vein. The arterial catheter was connected to a blood pressure transducer and systemic arterial blood pressure was monitored continuously. Buffered Evans blue solution $(0.5 \mathrm{ml})$ was then injected into the femoral vein. A burrhole was made over the left frontal cortex. A $10-\mu \mathrm{I}$ syringe ( $\mathrm{J}$ and $\mathrm{W}$ Scientific $\mathrm{Inc}$ ) with a deactivated fused silica needle (125 $\mu \mathrm{m}$ in diameter) was mounted on the arm of a stereotaxic frame. With the aid of magnification, the tip of the needle was manipulated $3.5 \mathrm{~mm}$ deep into the brain parenchyma and then withdrawn $1 \mathrm{~mm}$. The final depth into the parenchyma was therefore $2.5 \mathrm{~mm}$, corresponding to the gray-white matter interface.

Ten microliters of either saline solution, water, $6.5 \%$ methanol in saline, $\mathrm{LTB}_{4}, \mathrm{LTC}_{4}, \mathrm{LTC}_{4}, \mathrm{LTE}_{4}$, or arachidonic acid was injected into the parenchyma slowly over 1 hour. Five animals injected with arachidonic acid were given $\mathrm{BW} 755 \mathrm{C}(40 \mathrm{mg} / \mathrm{kg})$ intraperitoneally 1 hour prior to arachidonic acid injection, and 5 animals were given indomethacin $(6 \mathrm{mg} / \mathrm{kg})$ intraperitoneally 1 hour prior to arachidonic acid injection. Rats were decapitated 1 hour after the intraparenchymal injection and their brains were removed and frozen. The frozen brains were sliced coronally through the plane of injection. The surface of the slice was photographed and, using polar planimetry, the area of Evans blue extravasation was estimated as the percentage of total surface area.

Leukotrienes were supplied by Dr R. Rokach of MerckFrosst Inc, Canada. LTC $_{4}$ and $\mathrm{LTE}_{4}$ were received in water at a concentration of $0.2 \mathrm{mg} / \mathrm{ml}$. LTB $_{4}$ was received in $65 \%$ methanol in water at a concentration of $0.1 \mathrm{mg} / \mathrm{ml}$. Leukotrienes were subsequently diluted in saline. They were di-
From the Section of Neurosurgery, University of Michigan, Ann Arbor, MI 48109.
Received Oct 15, 1984, and in revised form Jan 21, 1985. Accepted for publication Jan 30, 1985 .

Address reprint requests to Dr Black. 


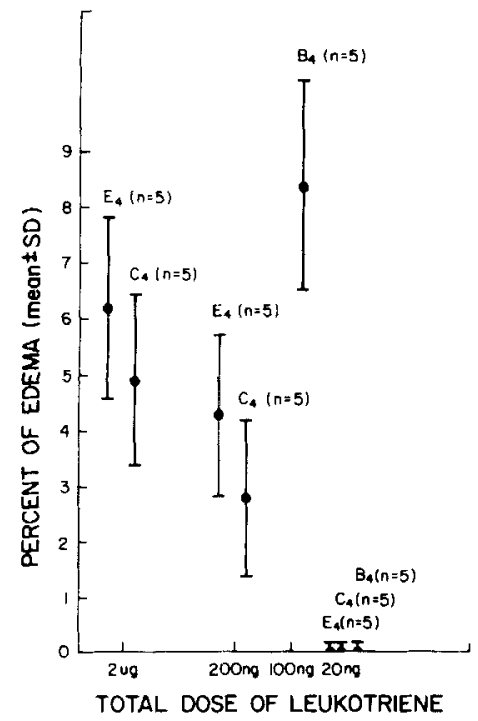

Fig 1. Percentage of area of Evans blue extravasation in a coronal section of brain centered on the injection site after a 10- $\mu$ l injection of leukotriene $B_{4}, C_{4}$, or $E_{4}$ over 1 bour.

vided into small aliquots and stored in glass vials that were vacuum sealed and frozen at $-70^{\circ} \mathrm{C}$. The total dose of $\mathrm{LTB}_{4}$ injected did not exceed $100 \mathrm{ng}$ per brain since the methanol content would then have been greater than $6.5 \%$. In preliminary studies we found that saline solutions with a methanol concentration greater than $6.5 \%$ produced some disruption in the $\mathrm{BBB}$. Indomethacin and free arachidonic acid were obtained from Sigma. Free arachidonic acid was dissolved in $0.3 \mathrm{M}$ sodium hydroxide and diluted in saline to a concentration of $1.0 \mathrm{mg} / \mathrm{ml}$. BW755C was a gift of Wellcome Research Laboratories, Kent, England. BW755C was dissolved in saline to a final concentration of $20 \mathrm{mg} / \mathrm{ml}$.

An analysis of variance was used for statistical analysis.

\section{Results}

Except for negligible blue staining along the insertion tract of the needle, none of the rats injected with saline or water had evidence of Evans blue extravasation. The percentage of blue area in rats injected with $6.5 \%$ methanol was also negligible $(0.68 \pm 0.21 \%$ of the area of the left hemisphere in the plane of injection). Animals injected with $\mathrm{LTB}_{4}, \mathrm{LTC}_{4}$, or $\mathrm{LTE}_{4}$ had significant extravasation of Evans blue in the area of injection (Fig 1).

Arachidonic acid injection also resulted in extravasation of Evans blue. This effect was, however, prevented by lipoxygenase inhibition with $\mathrm{BW} 755 \mathrm{C}$, but not by indomethacin (Figs 2, 3).

Blood pressure remained within physiological limits in all groups.

\section{Discussion}

Moskowitz and colleagues [7] reported increased levels of leukotrienes after cerebral ischemia and reperfusion in the gerbil brain and suggested that cortical

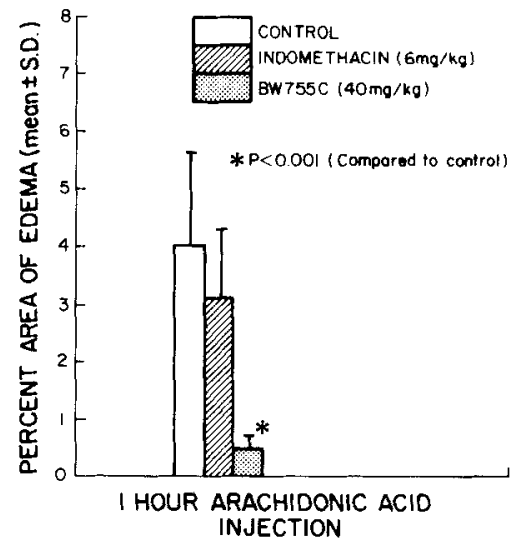

Fig 2. Percentage of area of Evans blue extravasation after a $10-\mu l$ injection of arachidonic acid ( $10 \mu \mathrm{g}$ total dose) over 1 bour. Five animals were pretreated with indometbacin and $S$ were pretreated with BW755C.

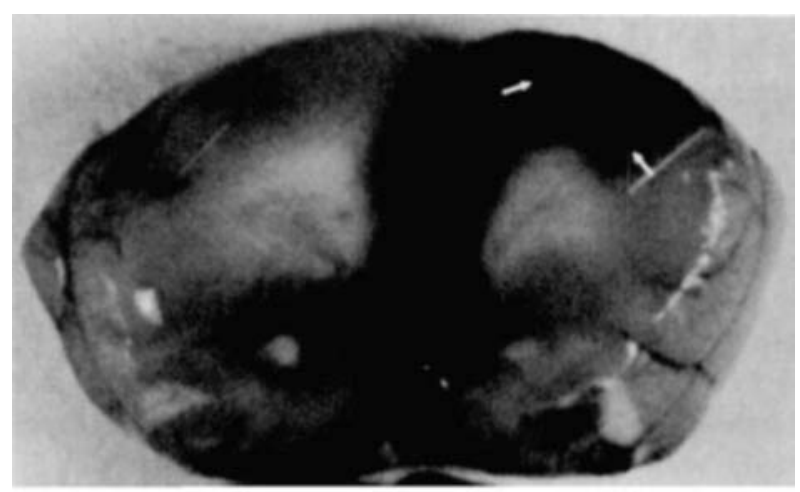

A

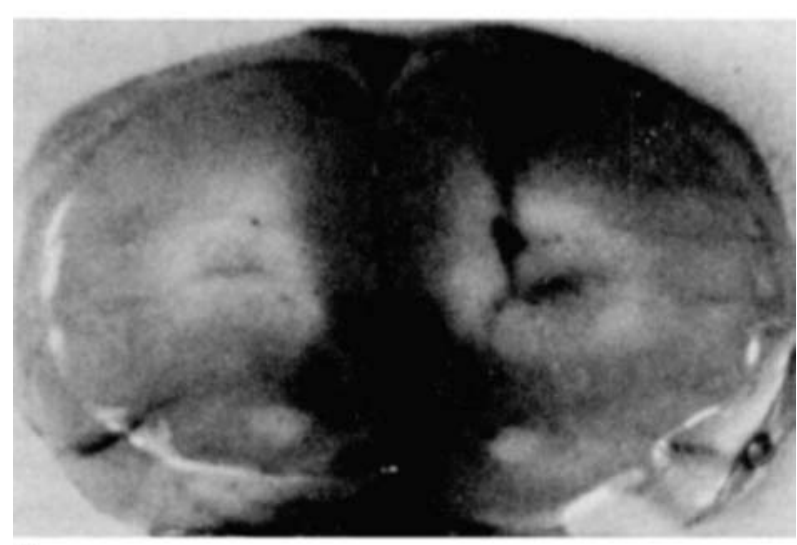

B

Fig 3. Coronal section through the plane of aracbidonic acid injection after $(A)$ pretreatment with indomethacin and $(B)$ pretreatment with $B W 755 C$. The amount of Evans blue extravasation seen in the left bemisphere (arrows in $A$ ) is markedly decreased after pretreatment with BW755C. 
gray matter was the most likely source of leukotriene synthesis. Since leukotrienes are potent vasoconstrictors [11] and they increase vascular permeability [2, 10], some investigators suspect that leukotrienes are involved in the pathogenesis of brain damage following cerebral ischemia $[2,7,11]$.

Our study demonstrates that $\mathrm{LTB}_{4}, \mathrm{LTC}_{4}$, or $\mathrm{LTE}_{4}$ (>20 ng) injected directly into brain parenchyma significantly increases $\mathrm{BBB}$ permeability. This effect is lost when the total local dose is less than $20 \mathrm{ng}$. The amount of leukotriene required to produce vasogenic edema by injection into the brain is, therefore, several orders of magnitude higher than that present in the cortex after ischemia-reperfusion experiments. The discrepancy may be attributed to: (1) leukotrienes have a short half-life; the baseline level of leukotriene during the 1-hour injection may be lower than the actual dose injected; (2) a longer time interval of exposure may be required for smaller doses of leukotrienes to increase BBB permeability; and (3) measurement of leukotrienes in lesions that primarily cause the vasogenic form of edema may reveal leukotriene levels higher than those in ischemic edema. Studies to determine whether a lipoxygenase inhibitor will decrease vasogenic edema in these conditions are warranted.

Other investigators have shown that arachidonic acid produces cerebral edema. The induction of brain edema by arachidonic acid is dose dependent and the resulting edema becomes maximal between 24 and 48 hours. The edema is reduced by pretreatment with dexamethasone but not by indomethacin [5]. Interestingly, dexamethasone is reported to decrease leukotriene synthesis [4].

In the present study the lipoxygenase inhibitor BW755C prevented the vasogenic edema normally caused by arachidonic acid. BW755C has also been shown to inhibit cyclooxygenase [8]. The protective effect of BW755C is unlikely to be related to its effect on cyclooxygenase, since indomethacin, at a dose reported to inhibit cyclooxygenase [1], did not provide a similar protective effect. Our findings suggest that the production of vasogenic edema by arachidonic acid is dependent on products of the 5-lipoxygenase enzyme, i.e., leukotrienes, rather than the products of cyclooxygenase.

Caronna and co-workers [5] suggested that the ability of arachidonic acid to produce brain edema is not specific. They also reported increased edema after injections of other polyunsaturated fatty acids, but not saturated fatty acids. Homa and colleagues [6], how- ever, recently reported that polyunsaturated fatty acids, but not saturated fatty acids, stimulate the formation of lipoxygenase products from arachidonic acid. Theoretically, therefore, other polyunsaturated fatty acids might increase edema by increasing the conversion of arachidonic acid to leukotrienes.

In our study, $\mathrm{LTB}_{4}$ produced a greater increase in BBB permeability than the other leukotrienes. Whether this relates to the ability of $\mathrm{LTB}_{4}$ to act as a chemotactic factor attracting leukocytes, which in turn may produce more leukotrienes, is speculative.

We believe that the development of vasogenic cerebral edema is multifactorial and depends on the stimuli inducing the edema process. This study demonstrates that leukotrienes do induce vasogenic edema and that the ability of arachidonic acid to induce edema can be prevented by pretreatment with a lipoxygenase inhibitor.

\section{References}

1. Abdel-Halim MS, Sjoquist B, Anggard E: Inhibition of prostaglandin synthesis in the rat brain. Acta Pharmacol Toxicol (Copenh) 43:266-272, 1978

2. Black $\mathrm{KL}$ : Leukotriene $\mathrm{C}_{4}$ induces vasogenic cerebral edema in rats. Prostaglandins Leukotrienes Med 14:339-340, 1984

3. Black KL, Hoff JT, Radin NS, Deshmukh GD: Eicosapentaenoic acid: effect on brain prostaglandins, cerebral blood flow and edema in ischemic gerbils. Stroke 15:65-69, 1984

4. Blackwell GJ, Carnuccion R, DiRosa D, et al: Macrocortin: a polypeptide causing the anti-phospholipase effect of glucocorticoids. Nature 287:147-149, 1980

5. Caronna JJ, Chan PH, Fishman RA: Protective effects of corticosteroids on fatty acid-induced cerebral edema. Trans Am Neurol Assoc 105:200-203, 1980

6. Homa ST, Conroy DM, Smith AD: Unsaturated fatty acids stimulate the formation of lipoxygenase and cyclooxygenase products in rat spleen lymphocytes. Prostaglandins Leukotrienes Med 14:417-427, 1984

7. Moskowitz MA, Kiwak KJ, Hekimian K, Levine L: Synthesis of compounds with properties of leukotrienes $\mathrm{C}_{4}$ and $\mathrm{D}_{4}$ in gerbil brains after ischemia and reperfusion. Science 224:886-888, 1984

8. Randall RW, Eakins KE, Higgs GA, et al: Inhibition of arachidonic acid cyclo-oxygenase and lipoxygenase activities of leukocytes by indomethacin and compound BW755C. Agents ACtions 10:553-555, 1980

9. Robak J, Duniec $Z$ : The influence of some 3-amino-2pyrazoline derivatives on cyclooxygenase and lipoxidase activities. Biochem Pharmacol 31:1955-1959, 1983

10. Soter NA, Lewis RA, Corey EJ, Austen KF: Local effects of synthetic leukotrienes $\left(\mathrm{LTC}_{4}, \mathrm{LTD}_{4}, \mathrm{LTE}_{4}\right.$, and $\left.\mathrm{LTB}_{4}\right)$ in human skin. J Invest Dermatol 80:115-119, 1983

11. Tager P, DuBoulay GH, Aitken V, Boullin DJ: Leukotriene $\mathrm{D}_{4}$ and the cerebral vasculature in vivo and in vitro. Prostaglandins Leukotrienes Med 11:281-297, 1983 